\title{
Simple 60-GHz Radio-over-Fiber System with Transmission Distance Improvement Employing Signal-to-Signal Beat Noise Compensation
}

\author{
Chun-Ting Lin, Wei-Erh Chen, and Hou-Tzu Huang
}

\begin{abstract}
We propose a simple 60-GHz RoF system using one single-electrode Mach-Zehnder modulator (MZM). By choosing proper carrier frequencies of two MZM driving signals, fiber transmission distance can be extended up to $12 \mathrm{~km}$. Compared with conventional intensity modulation with direct detection using 60-GHz MZM or electro-absorption modulator (EAM), the proposed RoF system can support longer fiber transmission distance (up to $12 \mathrm{~km}$ ) and has less bandwidth requirement of optical orthogonal frequency-division multiplexing (OFDM) transmitter (34 GHz vs. $64 \mathrm{GHz})$. However, the generated 60-GHz OFDM signal will have beat noise after square-law photo-detection. In this paper, the beat noise is analytically analyzed. Hence, the beat noise mitigation algorithm with the beat noise re-construction technique is proposed and utilized to reduce beat-noise-induced interference and improve signalto-noise ratio (SNR). 60-GHz 13.75-Gbps quadrature phase shift keying (QPSK) and 20.625-Gbps 8-quadrature-amplitudemodulation (QAM) OFDM signals with 3-m wireless transmission are experimentally demonstrated and transmitted over $12-\mathrm{km}$ and 6-km single-mode fiber, respectively.
\end{abstract}

Index Terms-Radio-over-fiber (RoF), 60 Ghz, orthogonal frequency division multiplexing (OFDM).

\section{INTRODUCTION}

$\mathbf{W}$ ITH the continuously growing demand for wireless video-based interactive and multimedia data services, $60-\mathrm{GHz}$ millimeter-wave wireless systems have been viewed as a promising candidate for multi-gigabit-per-second wireless access due to $7-\mathrm{GHz}$ license-free band (i.e. 57-64 GHz). However, 60-GHz wireless signal suffer from high path loss. Hence, the cell size of $60-\mathrm{GHz}$ pico-cells is generally limited to $10 \mathrm{~m}$, which is ideal for in-building applications. Consequently, wireless access networks at $60-\mathrm{GHz}$ require numerous base stations (BSs) to connect the access points. Therefore, the radio-over-fiber (RoF) system approach, which distributes RF signals from a central station (CS) to multiple BS over an optical fiber, is a promising means of reducing the overall cost of $60-\mathrm{GHz}$ wireless access networks owing to its nearly unlimited bandwidth and extremely low propagation loss [1]-[6]. For certain applications such as in-building, it is imperative that the employed RoF links are as simple as possible to reduce cost, while providing the needed performance. Recently, we proposed simple $60-\mathrm{GHz}$ RoF systems

Manuscript received July 31, 2012; revised October 31, 2012.

The authors are with the Institute of Photonic Systems, National Chiao Tung University, 301, Gaofa 3rd., Guiren Township, Tainan County 711, Taiwan (e-mail: jinting@mail.nctu.edu.tw).

Digital Object Identifier 10.1109/JSAC.2013.SUP2.1213009 with one single-electrode Mach-Zehnder modulator (MZM), and their analysis have been presented in [7]. Compared with conventional optical double sideband RoF systems using 60$\mathrm{GHz}$ MZM or electro absorption modulator [8], the proposed RoF system can support longer fiber transmission distance (4 km vs. $500 \mathrm{~m}$ ) and has less bandwidth requirement of optical orthogonal frequency-division multiplexing (OFDM) transmitter $(35.5 \mathrm{GHz}$ vs. $64 \mathrm{GHz})$. In this paper, we further improve the fiber transmission distance from $4 \mathrm{~km}$ to $12 \mathrm{~km}$ by choosing proper carrier frequencies of two MZM driving signals. However, the generated $60 \mathrm{GHz}$ OFDM signal will have beat noise after square-law photo-detection. Moreover, the beat noise is analytically analyzed, and the beat noise re-construction technique is derived. Hence, the beat noise mitigation algorithm with the beat noise re-construction technique is utilized to reduce beat-noise-induced interference and improve signal-to-noise ratio (SNR). 60-GHz 13. 75-Gbps quadrature phase shift keying (QPSK) and 20.625-Gbps 8quadrature-amplitude-modulation (QAM) OFDM signals with 3-m wireless transmission are experimentally demonstrated and transmitted over $12-\mathrm{km}$ and $6-\mathrm{km}$ single-mode fiber, respectively.

\section{Concept of Proposed System}

Fig. 1 illustrates the proposed simple 60-GHz RoF system using one single-electrode MZM biased at $V_{\pi}$. The MZM driving signal consists of two signals: an OFDM-modulated signal at a center frequency of $f_{1}$ and a sinusoidal signal at a frequency of $f_{2}$, as indicated in insets (I)-(II) of Fig. 1. Since the MZM is biased at the null point, the generated optical signal consists of two optical OFDM-modulated subcarriers and two un-modulated subcarriers and can be expressed as

$$
\begin{aligned}
E_{\text {out }}(t)= & E_{0} \cdot\left\{J_{0}\left(m_{2}\right) J_{1}\left(m_{1}\right) \cdot \cos \left[2 \pi\left(f_{c} \pm f_{1}\right) t\right]\right. \\
& \left.+J_{0}\left(m_{1}\right) J_{1}\left(m_{2}\right) \cdot \cos \left[2 \pi\left(f_{c} \pm f_{2}\right) t\right]\right\}
\end{aligned}
$$

where $f_{c}$ is the carrier frequency of the laser, $m_{1}$ and $m_{2}$ are the modulation indices defined as $\pi V_{1} / 2 V_{\pi}$ and $\pi V_{2} / 2 V_{\pi}$, respectively, and $V_{1}$ and $V_{2}$ are the amplitudes of OFDM and sinusoidal signals, respectively. $J_{n}()$ is the $n_{t h}$ order Bessel function of the first kind. For small modulation indices, we do not consider the magnitude of Bessel function with the higher order.

After square-law photo detection, the electrical signals are generated at the sum frequency $\left(f_{1}+f_{2}\right)$ and at the difference 

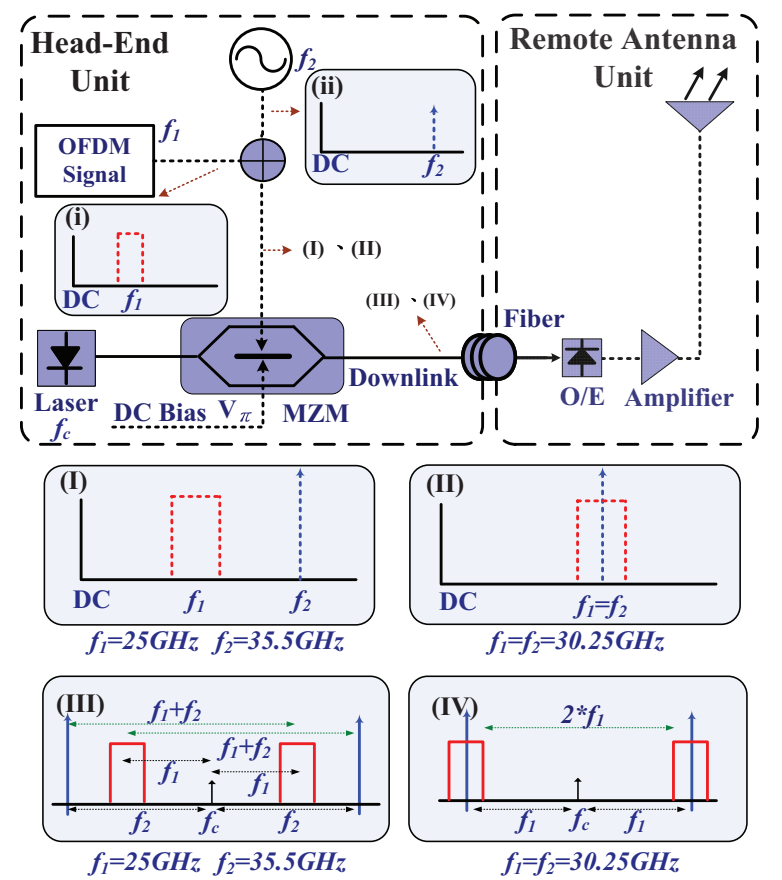

Fig. 1. The proposed simple RoF system using one single-electrode MZM.

frequency $\left(f_{2}-f_{1}\right)$. To reduce the bandwidth of the optical transmitter, the generated signal at the sum frequency is utilized for $60-\mathrm{GHz}$ wireless signals and can be expressed as

$$
\begin{array}{r}
i_{f_{1}+f_{2}}=R \cdot E_{0}^{2} J_{0}\left(m_{1}\right) J_{0}\left(m_{2}\right) J_{1}\left(m_{1}\right) J_{1}\left(m_{2}\right) \\
\cdot \cos 2 \pi\left(f_{1}+f_{2}\right) t
\end{array}
$$

where $R$ is the responsivity of photodiode. After transmission over standard single-mode fiber, the generated optical signal will have dispersion-induced phase shift on each of optical subcarriers. Hence, the generated electrical signal at the sum frequency can be rewritten as

$$
\begin{aligned}
& i_{f_{1}+f_{2}}=R \cdot E_{0}^{2} J_{0}\left(m_{1}\right) J_{0}\left(m_{2}\right) J_{1}\left(m_{1}\right) J_{1}\left(m_{2}\right) . \\
& \quad \cos \left[2 \pi^{2} \beta_{2}\left(f_{2}+f_{1}\right)\left(f_{2}-f_{1}\right) L\right] \cdot \cos 2 \pi\left(f_{1}+f_{2}\right) t
\end{aligned}
$$

where $L$ is the transmission distance and $\beta_{2}$ is chromatic dispersion parameter (17 ps/nm॰km). It can be seen that the power fading is observed due to fiber dispersion effect and is related to $\cos \left[2 \pi^{2} \beta_{2}\left(f_{2}+f_{1}\right)\left(f_{2}-f_{1}\right) L\right]$. Notably, the $\mathrm{RF}$ fading issue would become serious when the product of the sum frequency $\left(f_{2}+f_{1}\right)$ and the frequency difference $\left(f_{2}-f_{1}\right)$ increases. Hence, the allocation of the frequencies of $f_{1}$ and $f_{2}$ plays an important role on the RF power fading. For $60-\mathrm{GHz}$ wireless applications, the sum frequency is fixed at $60.5 \mathrm{GHz}$, and the frequency difference will dominate the performance of the RF fading. For the frequency difference of $10.5 \mathrm{GHz}$ case (i.e. $f_{1}=25 \mathrm{GHz}, f_{2}=35.5 \mathrm{GHz},\left(f_{2}-f_{1}\right)=10.5 \mathrm{GHz}$ ) as shown in insets (I) and (III) of Fig. 1, it can be seen that the power fading of the transmitted signal occurs periodically, as shown in Fig. 2. After 5.8-km and 17.3-km fiber transmission, the power penalty of the received signal will be larger than 30 $\mathrm{dB}$. As the frequency difference decreases, the fading issue can be mitigated. Finally, for the frequency difference of $0 \mathrm{GHz}$ case (i.e. $f_{1}=f_{2}=30.25 \mathrm{GHz}, f_{2}-f_{1}=0 \mathrm{GHz}$ ) as shown

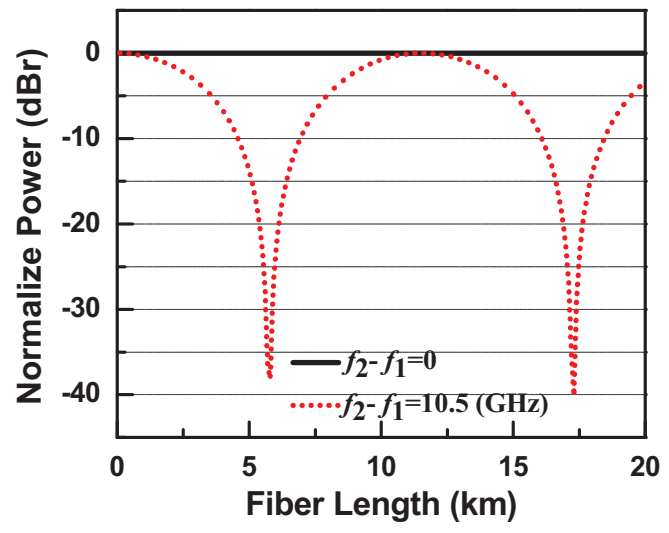

Fig. 2. Simulated RF power fading for different frequencies (i.e. $f_{1}-f_{2}$ ) versus fiber transmission length.

in insets (II) and (IV) of Fig. 1, the system will have no power penalty after the fiber transmission.

To consider the full $7 \mathrm{GHz}$ bandwidth at $60 \mathrm{GHz}$, an OFDM driving signal is up-converted to a center frequency of $f_{1}$. After square-law photo detection, the generated OFDM signal will occupy at a center frequency of $60.5 \mathrm{GHz}$ (i.e. 57-64 GHz). Fig. 3 shows the RF spectra of OFDM signals from $57-64 \mathrm{GHz}$ after transmission of different fiber lengths. For the case of $f_{2}-f_{1}=10.5 \mathrm{GHz}$, the RF fading is negligible over the 7-GHz spectrum after 1-km transmission. Beyond 1$\mathrm{km}$ transmission, the spectrum in the lower frequency begins to experience power attenuation because they are generated from larger frequency differences. After $3-\mathrm{km}$ and $5-\mathrm{km}$ transmission, the power fadings are less than $-10 \mathrm{~dB}$ and more than $-30 \mathrm{~dB}$, respectively. For the case of $f_{2}-f_{1}=0 \mathrm{GHz}$, the transmission distance can be improved as shown in Fig. 3 (b). The power fading is less than $-10 \mathrm{~dB}$ as the transmission distance is up to $10 \mathrm{~km}$. Note that the simulation in Fig. 2 only shows the power fading result of two single tones. However, one of the two single tones has OFDM signals with $7-\mathrm{GHz}$ bandwidth. That's why the RF power fading still occurs in Fig. 3 (b), and the spectrum at the higher frequency experiences higher power fading.

However, if the frequency difference $\left(f_{2}-f_{1}\right)$ is less than $10.5 \mathrm{GHz}$, the beat term of two optical OFDM-modulated subcarriers at the frequencies of $\left(f_{c}+f_{1}\right)$ and $\left(f_{c}-f_{1}\right)$ will generate electrical beat noise occupied at the desired $60-\mathrm{GHz}$ signal band $(57-64 \mathrm{GHz})$ after photo-detection, as shown in Fig. 4. As the frequency difference is equal to $10.5 \mathrm{GHz}$, i.e. $f_{1}=25 \mathrm{GHz}$ and $f_{2}=35.5 \mathrm{GHz}$, the beat noise falls just out of the 60-GHz OFDM signal band. Although the fading issue can be completely mitigated with $f_{1}=f_{2}=30.25 \mathrm{GHz}$, the generated electrical $60-\mathrm{GHz}$ signal has more beat noise as shown in Fig. 4(b). Therefore, there are tradeoffs between RF fading and beat noise interference.

\section{Mathematical Model of Beat Noise}

To extend the effective fiber transmission distance, we choose $f_{1}=f_{2}=30.25 \mathrm{GHz}$. Thus, the beat noise will affect the system performance significantly. To mitigate the penalty caused by beat noise, we investigate the model of the 
(a)

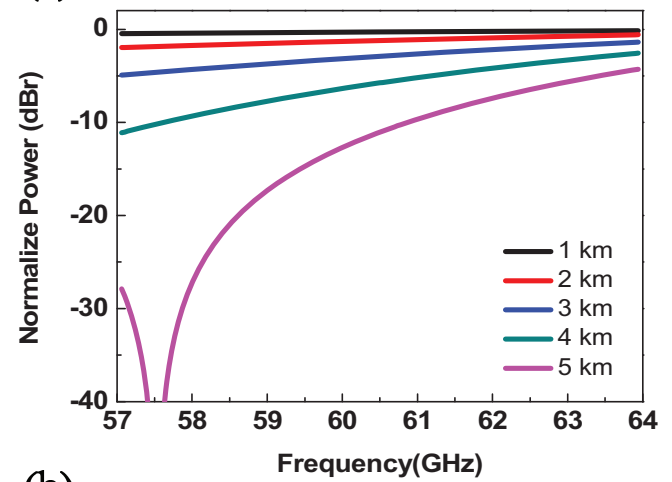

(b)

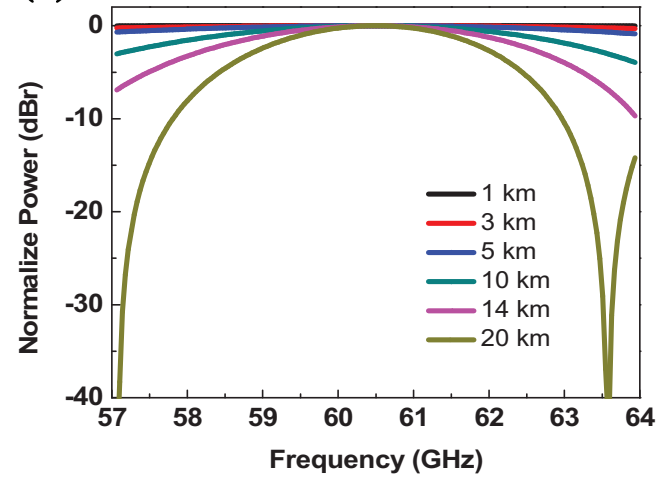

Fig. 3. Simulation results of RF fading at $60-\mathrm{GHz}$ band versus different fiber lengths: (a) $f_{1}=25 \mathrm{GHz}$ and $f_{2}=35.5 \mathrm{GHz}$; (b) $f_{1}=f_{2}=30.25$ $\mathrm{GHz}$.

beat noise and then mitigate the effect of that. The electrical OFDM signal for driving the MZM can be described as

$$
\begin{aligned}
& E(t)=R E\left\{A e^{j 2 \pi f_{2} t}+\sum_{k=-\frac{N}{2}, k \neq 0}^{\frac{N}{2}} d(k) e^{j 2 \pi\left(\frac{k}{N}+f_{1}\right) t}\right\} \\
& =R E\left\{A e^{j 2 \pi f_{2} t}+\sum_{k=-\frac{N}{2}, k \neq 0}^{\frac{N}{2}} d_{r}(k) e^{j\left[2 \pi\left(\frac{k}{N}+f_{1}\right) t+\varphi_{k}\right]}\right\}
\end{aligned}
$$

where the first and second terms of Eq. (5) are the sinusoidal signal and the OFDM signal at the center frequencies of $f_{2}$ and $f_{1}$, respectively. $N$ is fast Fourier transform (FFT) length, $d(k)$ is the complex data of the $k^{t h}$ OFDM subcarrier, and $d_{r}$ and $\varphi_{k}$ are the amplitude and phase information of the OFDM subcarriers, respectively. The optical field at the input of the $\mathrm{MZM}$ is given by

$$
E_{\text {in }}=E_{0} e^{j 2 \pi f_{c} t}
$$

Since the single-electrode MZM is biased at the null point for the proposed system, the output optical signal can be derived as

$$
\begin{gathered}
E_{\text {out }}(t)=\frac{1}{\sqrt{2}} E_{0} e^{j 2 \pi f_{c} t}\left\{e^{j \frac{\pi}{2 V_{\pi}}\left[V_{\pi}+E(t)\right]}+e^{j \frac{\pi}{2 V_{\pi}}\left[V_{\pi}+E(t)\right]}\right\} \\
=\frac{1}{\sqrt{2}} E_{\text {in }} \cos \left[\frac{\pi}{2 V_{\pi}} E(t)+\frac{\pi}{2}\right]
\end{gathered}
$$

Under the condition of the small modulation index, the Bessel function with the order of more than 2 can be ignored, and the
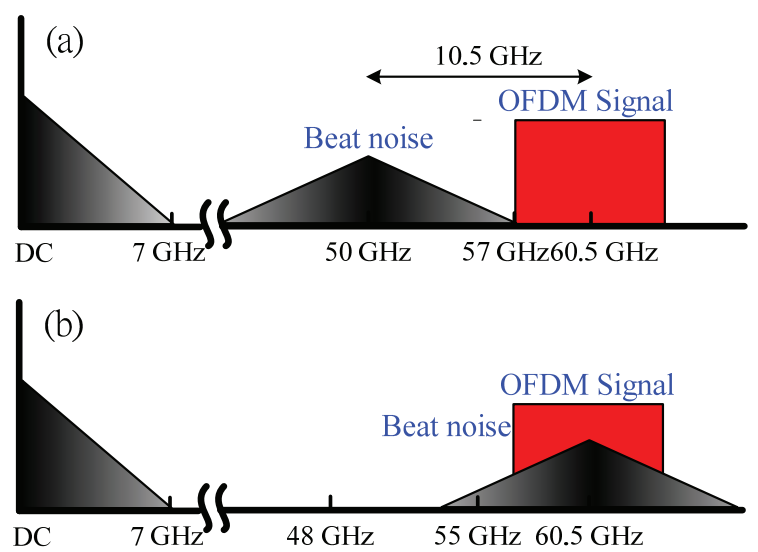

Fig. 4. Beat noise interference in the proposed system: (a) $f_{1}=25 \mathrm{GHz}$ and $f_{2}=35.5 \mathrm{GHz}$; (b) $f_{1}=f_{2}=30.25 \mathrm{GHz}$.

output optical field can be simplified as (9), where $m^{\prime} \equiv \frac{A \pi}{2 V_{\pi}}$ and $m q \equiv \frac{d_{r}(q) \pi}{2 V_{\pi}}, q=n$ or $k$. With the condition of $f_{1}=$ $f_{2}=30.25 \mathrm{GHz}$, we can further simplify the equation where (10) and (11) are the upper and lower side band of the optical signal, respectively. $H^{\prime}$ and $H(k)$ are rewritten as:

$$
\begin{gathered}
H^{\prime}=J_{1}\left(m^{\prime}\right) \prod_{n=-\frac{N}{2}, k \neq 0}^{\frac{N}{2}} J_{0}\left(m_{k}\right) \\
H(k)=J_{0}\left(m^{\prime}\right) \sum_{k=-\frac{N}{2}, k \neq 0}^{\frac{N}{2}}\left[\prod_{n \neq k, n \neq 0, n=-\frac{N}{2}}^{\frac{N}{2}} J_{0}\left(m_{n}\right)\right] J_{1}\left(m_{k}\right)
\end{gathered}
$$

After the photodiode, the converted electrical signal can be expressed as (14)-(17) at the top of the next page, where (14) is the DC signals, (15) is the self-induced beat noise, and (16) is the desired OFDM signal. The performance is determined by the power ratio between the desired OFDM signal and beat noise, which depends on $H(k)$ and $H^{\prime}$. Since $H(k)$ and $H^{\prime}$ are related to the amplitudes of electrical OFDM and sinusoidal driving signals, we can demodulate the received OFDM signal by choosing the power ratio between electrical sinusoidal wave and OFDM signal properly with the reasonable beat noise effect and other noise.

To further reduce the beat noise effect, the beat noise mitigation technique is utilized, shown as in Fig. 5 [9]. The demodulation of the OFDM signal includes CP removal, FFT, one-tap channel equalization, and hard decision. We need to feedback the hard decision signal to remove the beat noise, and the feedback processing follows these steps: 1) Reconstruction of the received signal by hard decision and channel de-equalization. 2) Cancellation of the beat noise term. 3) Re-demodulation of OFDM signals. For equalizer, the even subcarriers of chaining symbol are set null. After square-law photo detection, the SSBI will only occur in even subcarriers. Thus, we can obtain the one-tap equalizer of even subcarriers without SSBI. Furthermore, the equalizer of odd subcarriers is calculated from the interpolation of even subcarriers [9]. 


$$
\begin{aligned}
E_{\text {out }}(t)= & \frac{1}{\sqrt{2}} E_{\text {in }}\left\{2 J_{0}\left(m^{\prime}\right) \sum_{k=-\frac{N}{2}, k \neq 0}^{\frac{N}{2}}\left[\prod_{n=-\frac{N}{2}, n \neq 0, n \neq k}^{\frac{N}{2}} J_{0}\left(m_{n}\right)\right] J_{1}\left(m_{k}\right)\right. \\
& \left.\cdot \cos \left[2 \pi\left(\frac{k}{N}+f_{1}\right) t+\varphi_{k}\right]+2 J_{1}\left(m^{\prime}\right) \cos \left(2 \pi f_{2} t\right) \prod_{n=-\frac{N}{2}, n \neq 0}^{\frac{N}{2}} J_{0}\left(m_{k}\right)\right\}
\end{aligned}
$$

$$
\begin{aligned}
E_{\text {out }}= & E_{0}\left\{H^{\prime} e^{j 2 \pi\left(f_{c}+f_{1}\right) t}+\sum_{k=-\frac{N}{2}, k \neq 0}^{\frac{N}{2}} H(k) e^{j\left[2 \pi\left(f_{c}+\frac{k}{N}+f_{1}\right) t+\varphi_{k}\right]}\right\} \\
+ & E_{0}\left\{H^{\prime} e^{j 2 \pi\left(f_{c}+f_{1}\right) t}+\sum_{k=-\frac{N}{2}, k \neq 0}^{\frac{N}{2}} H(k) e^{j\left[2 \pi\left(f_{c}-\frac{k}{N}-f_{1}\right) t-\varphi_{k}\right]}\right\}
\end{aligned}
$$

$$
\begin{gathered}
I_{\text {current }}=R\left|E_{\text {out }}\right|^{2}=R\left\{\sum_{k=0}^{N-1} 2|H(k)|^{2}+2\left|H^{\prime}\right|^{2}\right. \\
+R E\left\{2 R \sum_{k=-\frac{N}{2}, k \neq 0}^{\frac{N}{2}} \sum_{l=-\frac{N}{2}, l \neq 0}^{\frac{N}{2}} H(k) H^{*}(l) e^{j\left\{2 \pi\left[\frac{(k+l)}{N}+2 f_{1}\right] t+\left[\varphi_{k}+\varphi_{l}\right]\right\}}\right\} \\
+R E\left\{2 R \sum_{k=-\frac{N}{2}, k \neq 0}^{\frac{N}{2}}\left(H^{*}(k) H^{\prime}+H(k) H^{* \prime}\right) e^{j\left[2 \pi\left(\frac{k}{N}+2 f_{1}\right) t+\varphi_{k}\right]}\right\} \\
\left.+R E\left\{2 R H^{\prime} H^{* \prime} e^{j 2 \pi\left(2 f_{1}\right) t}\right\}\right\}
\end{gathered}
$$

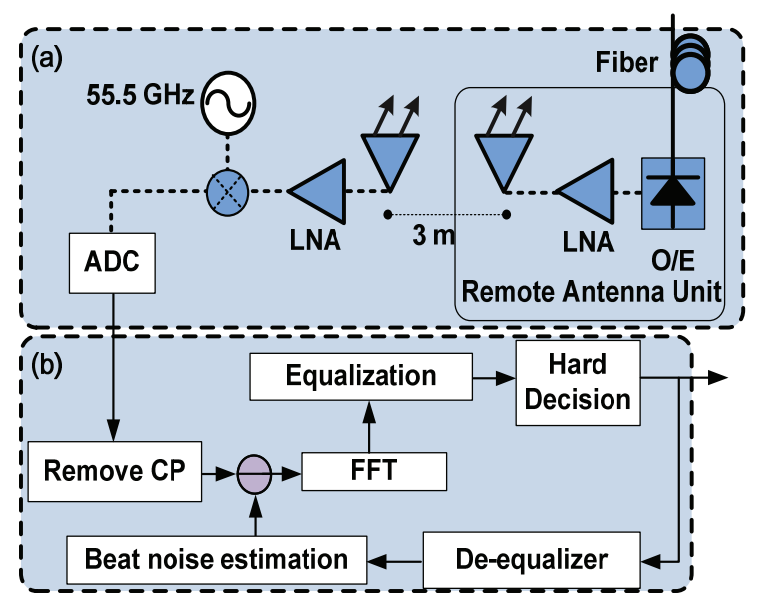

Fig. 5. The depiction of (a) wireless end, and (b) the SSBI mitigation algorithm.

\section{EXPERIMENTAL RESULTS}

Fig. 6 shows the experimental setup which utilizes a singleelectrode MZM biased at the null point. The electrical driving signal consists of an OFDM signal at a center frequency of $30.5 \mathrm{GHz}$ and a sinusoidal signal at $30.5 \mathrm{GHz}$. The baseband
OFDM signal is generated by arbitrary waveform generator. The sampling rate is $10 \mathrm{GSample/s}$. The inverse fast Fourier transform (IFFT) length is 256, resulting in a subcarrier symbol rate of $39.0625 \mathrm{MSymbol} / \mathrm{s}$. 176 subcarriers of OFDM signals are modulated with QPSK or 8-QAM format. Then, the baseband OFDM signal is up-converted with I/Q mixer. Hence, the OFDM signal occupies $7-\mathrm{GHz}$ bandwidth at a center frequency of $30.5 \mathrm{GHz}$, and the data rate are 13.75 Gbps and 20.625 Gbps for QPSK and 8-QAM OFDM signals, respectively. At the output of the single-electrode MZM, the optical OFDM signal comprises two optical sidebands with a frequency difference of $60.5 \mathrm{GHz}$. Note that the bandwidth of optical transmitter is only $34 \mathrm{GHz}$. At the receiver, the generated 60-GHz OFDM signal after 3-m wireless transmission is down-converted to $5 \mathrm{GHz}$ and captured by a digital oscilloscope with a 40-GSample/s sampling rate and a 3-dB bandwidth of $16 \mathrm{GHz}$.

For direct-detection optical $60-\mathrm{GHz}$ OFDM signals, the power ratio between the optical RF carrier and the optical OFDM signal is one of key parameters to the performance. When the generated $60-\mathrm{GHz}$ OFDM signals have beat noise interference which is generated from the beat term of two optical OFDM-modulated subcarriers after photo detection, the power ratio plays more important role on the performance. Hence, we define the electrical carrier-to-signal power ratio (CSPR) of the RF driving signal to the OFDM driving signal. 


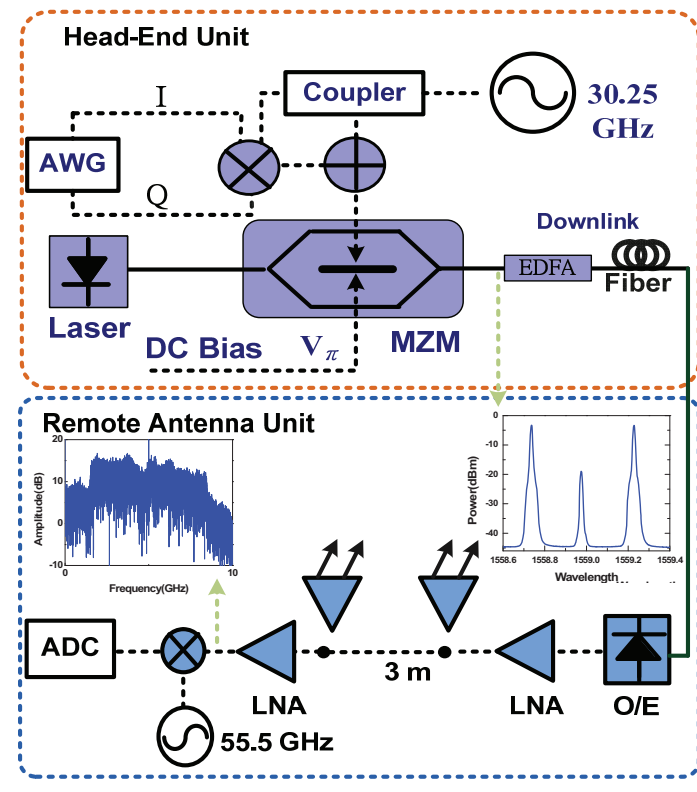

Fig. 6. Experimental setup of the proposed RoF system.
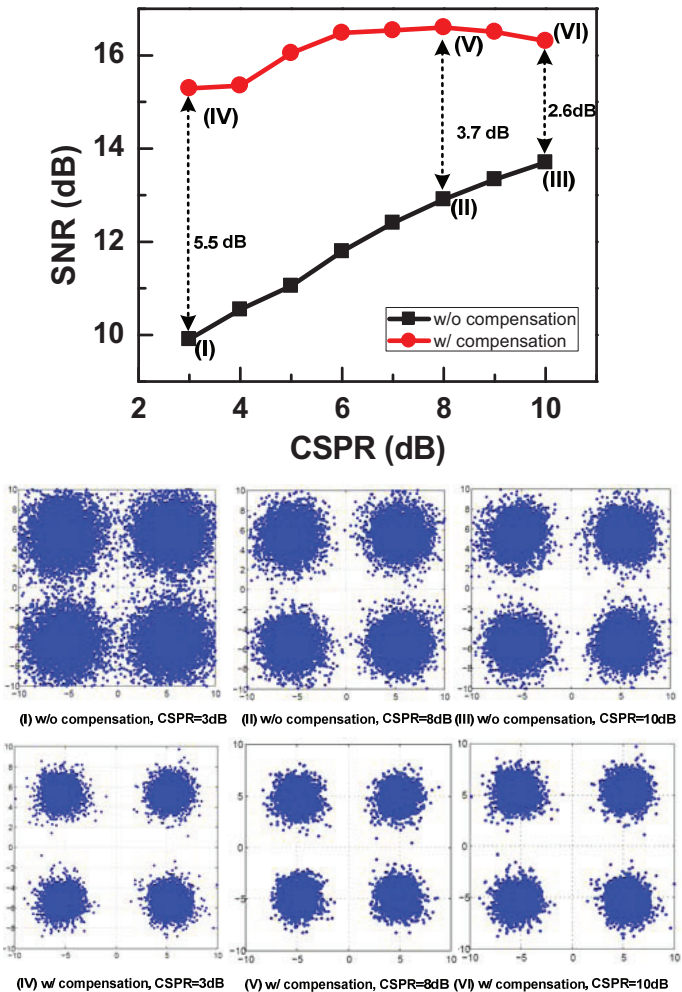

Fig. 7. The average SNRs of 60-GHz QPSK OFDM subcarriers versus CSPR. Optical received power is $-2 \mathrm{dBm}$.

Fig. 7 shows the SNR versus the different CSPR with constellations for the back-to-back (BTB) case. Without the beat noise mitigation, the SNR increases as CSPR value increases. This is because the power of beat noise decreases as CSPR increases. After the beat noise mitigation, it can be seen that the constellations of QPSK signals are obviously clearer and (a)

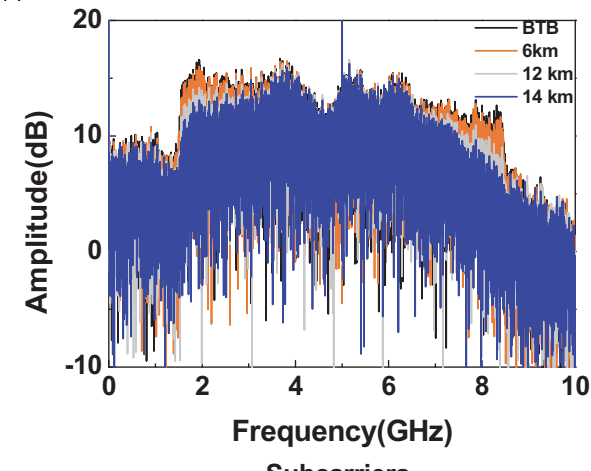

(b)

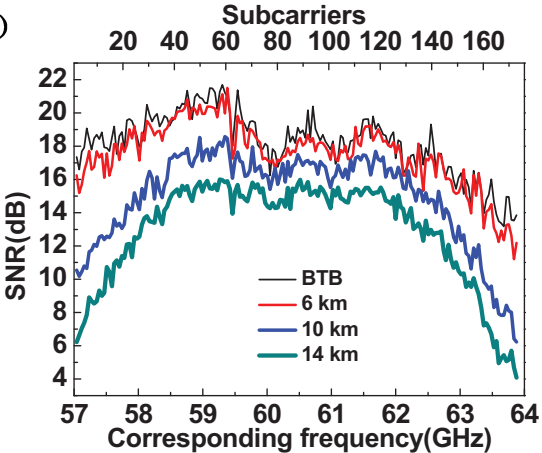

Fig. 8. (a) The down-converted electrical spectra for different standard fiber transmission lengths; (b) SNR versus different subcarrier for different fiber transmission lengths.

(a)

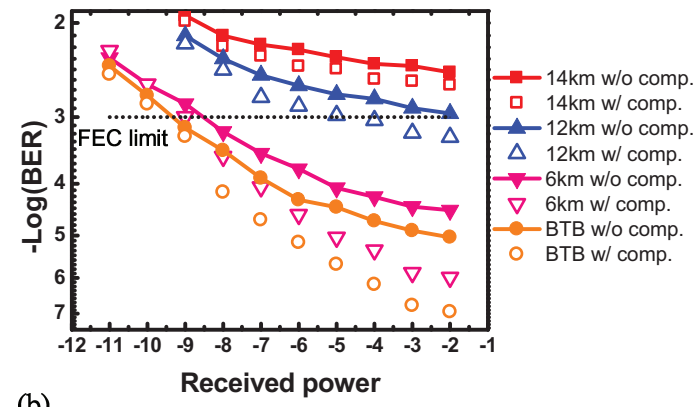

(b)

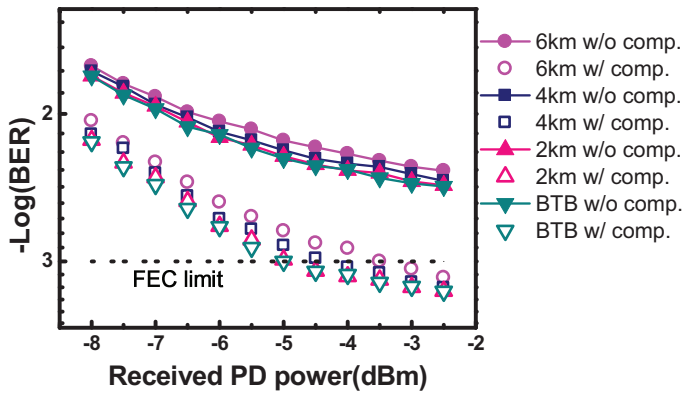

Fig. 9. (a) BER curves of QPSK OFDM signals including different fiber lengths and 3-m wireless distance; (b) BER curves of 8QAM OFDM signals including different fiber lengths and 3-m distance.

the SNR increases. There are 5.5-dB, 3.7-dB, and 2.6-dB SNR improvements after beat noise mitigation as CSPRs are $3 \mathrm{~dB}$, $8 \mathrm{~dB}$ and $10 \mathrm{~dB}$, respectively. Notably, with the beat noise mitigation, the SNR value can reach the maximum value at the optimal CSPR of $8 \mathrm{~dB}$ due to the tradeoff between the residual beat noise interference and the noise. The SNR performance is 


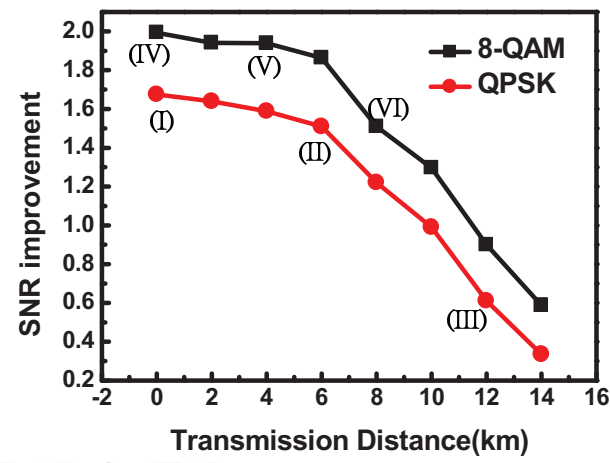

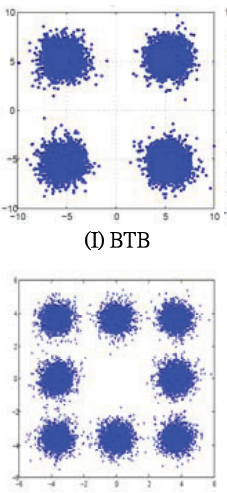

(IV) BTB

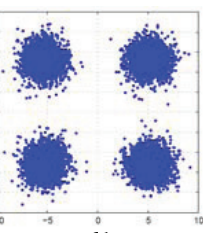

(II) $6 \mathrm{~km}$

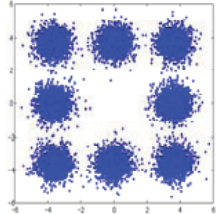

(V) $4 \mathrm{~km}$

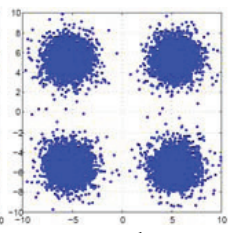

(III) $12 \mathrm{~km}$

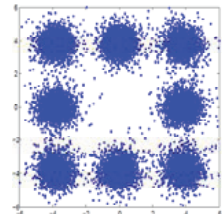

(VI) $8 \mathrm{~km}$
Fig. 10. The average SNR improvement versus fiber transmission distance.

dominated by the residual beat noise and the system noise at the lower and higher CSPR, respectively. As mentioned in the theoretical simulation for the case of $f_{2}=f_{1}=30.25 \mathrm{GHz}$, $7 \mathrm{GHz}$-wide OFDM signal will start to have power fading at lower and higher frequency as the fiber transmission distance increases as shown in Fig. 3(b). Fig. 8(a) shows the downconverted electrical spectra for different standard single-mode fiber transmission lengths. The OFDM signals suffer from power fading more seriously at lower and higher frequencies, resulting in lower SNRs at those frequencies. Note that the spectrum at the high frequency has more power fading, which is identical to the theoretical simulation.

Figs. 9(a) and 9(b) shows the experimental result of BER curves versus received power for QPSK and 8-QAM format, respectively. Note that the BER was converted from EVM by the relation equation of EVM and BER [11]. After 6-km fiber transmission with beat noise compensation, the power penalty at BER of $10^{-3}$ is less than $1 \mathrm{~dB}$ for the QPSK format. The effective fiber transmission distance with the beat noise mitigation can reach $12 \mathrm{~km}$ under FEC limit of $10^{-3}$. Compared with previous simple RoF system with the same modulation format [7], the proposed RoF system has 3 times of fiber transmission distance (i.e. $4 \mathrm{~km} \rightarrow 12 \mathrm{~km}$ ). As for 8QAM OFDM signals, the effective fiber transmission distance with the beat noise mitigation can reach $6 \mathrm{~km}$ under FEC limit of $10^{-3}$. Compared with the conventional optical DSB RoF system [8] with the same modulation format, the proposed RoF system has 12 times of fiber transmission distance (i.e. $500 \mathrm{~m} \rightarrow 6 \mathrm{~km})$.

Notably, the SNR improvement due to beat noise com- pensation decreases as the fiber transmission increases as shown in Fig. 10. The reason is that the RF fading increases and dominates the performance when the fiber transmission distance increases. Thus, the hard decision in the feedback loop, which is used to estimate the beat noise, will make a lot of wrong decisions. This result makes the mitigation technique not reliable.

\section{CONCLUSION}

We have theoretically and experimentally investigated the performance of the proposed simple RoF system for transporting and generating multi-Gbps OFDM-modulated wideband wireless signals at $60 \mathrm{GHz}$. The RoF system employs one single-electrode MZM. Theoretical analysis of the proposed system shows that it can achieve longer fiber transmission distances without any chromatic dispersion compensation by choosing appropriate input signal frequencies. The proposed RoF system with beat noise mitigation algorithm experimentally transported $13.75-\mathrm{Gb} / \mathrm{s}$ QPSK OFDM and 20.625-Gb/s 8-QAM OFDM signals at $60 \mathrm{GHz}$ over $12-\mathrm{km}$ and 6-km fiber distance, respectively.

\section{REFERENCES}

[1] Z. Cao, Z. Dong, J. Lu, M. Xia, and L. Chen, "Optical OFDM signal generation by optical phase modulator and its application in ROF system," in Proc. 35th European Conf. Optical Commun., 2009, pp. $20-24$.

[2] Z. Jia, J. Yu, G. Ellinas, and G. K. Chang, "Key enabling technologies for optical-wireless networks: Optical millimeter-wave generation, wavelength reuse and architecture," J. Lightw. Technol., vol. 25, no. 11, pp. 3452-3471, Nov. 2007.

[3] Z. Xu, X. Zhang, and J. Yu, "Frequency upconversion of multiple RF signals using optical carrier suppression for radio over fiber downlinks," Opt. Express, vol. 15, no. 25, pp. 16737-16737, 2007.

[4] M. Wei $\boldsymbol{\beta}$, A. Stöhr, M. Huchard, S. Fedderwitz, B. Charbonnier, V. Rymanov, S. Babiel, and D. Jäger, "60GHz radio-over-fibre wireless system for bridging 10Gb/s ethernet links," in Proc. 34th European Conf. Optical Commun., pp. 1-2.

[5] P. Smulders, "Exploiting the $60 \mathrm{GHz}$ band for local wireless multimedia access: Prospects and future directions," IEEE Commun. Mag., vol. 40, no. 1, pp. 140-147, Jan. 2002.

[6] M. Sauer, A. Kobyakov, and J. George, "Radio over fiber for picocellular network architectures," J. Lightw. Technol., vol. 25, no. 11, pp. 33013320, Nov. 2007.

[7] W. J. Jiang, C. T. Lin, A. Ng'oma, P. T. Shih, J. Chen, M. Sauer, F. Annunziata, and S. Chi, "Simple 14 Gbps short-range radio-overfiber system employing a single-electrode MZM for $60 \mathrm{GHz}$ wireless applications," J. Lightw. Technol., vol. 28, no. 16, pp. 2238-2246, Aug. 2010.

[8] A. Ng'oma, P. Shih, J. George, F. Annunziata, M. Sauer, C. Lin, W. Jiang, J. Chen, and S. Chi, "21 Gbps OFDM Wireless signal transmission at $60 \mathrm{GHz}$ using a simple IMDD radio-over-fiber system," in Proc. Optical Fiber Commun. Conf., pp. 1-3.

[9] W. R. Peng, B. Zhang, K. M. Feng, X. Wu, A. E. Willner, and S. Chi, "Spectrally efficient direct-detected OFDM transmission incorporating a tunable frequency gap and an iterative detection technique," J. Lightw. Technol., vol. 27, no. 24, pp. 5723-5735, Dec. 2009.

[10] Z. Cao, J. Yu, M. Xia, Q. Tang, Y. Gao, W. Wang, and L. Chen, "Reduction of intersubcarrier interference and frequency-selective fading in OFDM-ROF systems," J. Lightw. Technol., vol. 28, no. 16, pp. 24232429, Aug. 2010.

[11] R. Shafik, S. Rahman, and A. R. Islam, "On the extended relationships among EVM, BER and SNR as performance metrics," in Proc. IEEE ICECE, Dec. 2006, pp. 408-411. 


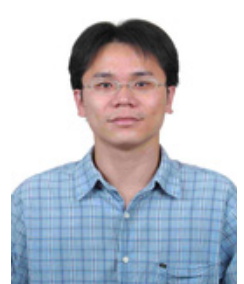

Chun-Ting Lin received the B.S. and M.S. degrees in materials science and engineering from National Tsing Huang University (NCTU), Hsinchu, Taiwan, in 1997 and 2001, respectively, and the Ph.D. degree in electro-optical engineering from NCTU in 2007. From 2007 to 2009, he was a Research Associate with the Department of Photonics, National Chiao Tung University. In 2009, he joined the faculty of NCTU, where he is currently an Assoicate Professor with the Institute of Photonic Systems. His research interests are radio-over-fiber systems, optical millimeter/sub-terahertz wave generation and applications, optical data formats, and opto-electronic packages.

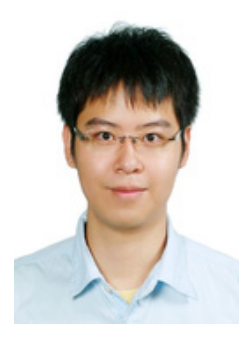

Wei-Erh Chen received the B.S. degree in materials science and engineering from National Tsing Huang University (NCTU), Hsinchu, Taiwan, in 2010 and the M.S. degree in photonic systems from NCTU in 2012. His current research interests include optical fiber communication, silicon-photonics, optoelectronic materials, and high-speed semiconductor devices.

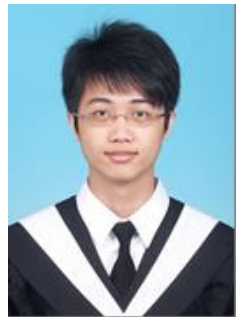

Hou-Tzu Huang received the B.S. degree from the Department of Photonics, National Chiao Tung University (NCTU), Hsinchu, Taiwan, in 2012. He is currently a Ph.D. student in the Institute of Photonics Systems at NCTU. 